\title{
Flotation of hard coal in saline water
}

\author{
Anna Mlynarczykowska ${ }^{1, a}$, Agnieszka Nowak $^{1}$, and Klaudia Tupek ${ }^{1}$ \\ ${ }^{1}$ AGH University of Science and Technology, Faculty of Mining and Geoengineering, Department of Environmental \\ Engineering and Mineral Processing, Poland
}

\begin{abstract}
With the use of saline process water, the ash content in the concentrate was $6 \%$ and in the tailings $70 \%$. Of course the upgrading plants which apply flotation for coal slurries still use mine water in technological processes. The water quality, particularly the content and type of salts in water, fundamentally influences the surface properties of the solid, gaseous and liquid phases, which remain in contact during the process. The properties of these phases and interactions between them determine the probability of occurrence of elementary flotation actions that is collision, adhesion and separation, and ultimately the upgrading process effectiveness expressed by technological parameters. The paper presents the results of flotation effectiveness analysis for coal as a model raw material. The object of evaluation was qualitative parameters of the upgrading process products on the basis of flotation tests conducted for variable pulp salinity. The principal goal was to determine the impact of process water quality on the course and the effectiveness of flotation.
\end{abstract}

\section{Introduction}

Hard coal is the main source of energy in Poland and its properties result from the condition of formation. The mineral matter of raw coal consists of hydrophilic minerals, mainly clays, quartz, carbonate minerals such as calcite and dolomite, gypsum and pyrite. Flotation is not the basic coal upgrading technology, but it is useful in pyrite removal and is used to reduce the ash content in the concentrate. The use of surfactants in the coal flotation is justified from the physical and chemical point of view. One must also remember that under industrial conditions flotation is performed with the mine water, which contains various well soluble inorganic salts. The types and amounts of salts depend on the mining conditions. The presence of salt in the flotation environment affects the action of flotation reagents, and consequently the process results and quality of concentrate. The use of process water in coal upgrading in processing plants began as early as in the 1980's. However, the main reason to study it was treatment of small coal particles and regulation of water and slurry management in the plants in terms of solids content in concentrators and the quality of concentrate. Since the observation that presence of salts, mostly inorganic, improves the flotation of naturally hydrophobic particles, many theories have been formulated to explain this phenomenon $[1,2,3]$. The research conducted by showed that the flotation recovery reached its maximum at the minimum value of zeta potential. However, Harvey et al. [4] proved that the improvement of coal flotability in the

${ }^{a}$ Corresponding author: mindziu@agh.edu.pl 
solutions of electrolytes cannot be explained exclusively by reduction of the zeta potential. Ozdemir et al. [5] revealed that the coal flotation results in brine $(1 \mathrm{M} \mathrm{NaCl})$ cannot be fully explained by the physical and chemical surface properties, particularly because the wetting angle for coal in the saline water is the same as in the demineralized water. Hence, explicit determination of mechanisms which improve the coal flotability under conditions of significant salinity is still an open question.

\section{Experimental}

\subsection{Material}

The feed used in flotation processes was grade 33 hard coal from one of Polish mines. The material preparation included comminution to obtain the particle size below $0.5 \mathrm{~mm}$. The wet sieve analysis was performed and the following particle size classes were obtained: $0.5-0.4,0.4-0.315,0.315-0.2$, $0.2-0.1,0.1-0.063 \mathrm{~mm}$ which were tested for the ash content. The tested material was kept in vacuum to prevent coal surface oxidation. All particle size classes were used in the flotation tests, the impact of particle size and density on the upgrading results in the salt-free flotation environment were analysed. On the basis of the results, two particle size classes $0.315-0.2$ and $0.1-0.063 \mathrm{~mm}$ were selected for flotation tests under conditions of various pulp salinity. The flotation tests were also conducted for the particle size below $0.5 \mathrm{~mm}$.

\subsection{Flotation Experiment}

The flotation tests were performed in the Denver pneumatic-mechanical laboratory machine. The work space volume was $1 \mathrm{dm}^{3}$, rotor speed was constant $1850 \mathrm{rpm}$, and the pulp density was $70 \mathrm{~g} / \mathrm{dm}^{3}$. Using low flotation pulp densities allows to achieve better flotation upgrading results, and the constant rotor speed guarantees the unchanging amount and size of air bubbles [6].

Individual flotation tests for selected narrow grain size classes were performed in distilled water, saline mine water and in brines made by adding $\mathrm{NaCl}$ to the mine water. The content of chlorine ions $\left(\mathrm{Cl}^{-}\right)$in saline water was $255.6 \mathrm{mg} / \mathrm{dm}^{3}, 23.4 \mathrm{~g} / \mathrm{dm}^{3}$ and $52.6 \mathrm{~g} / \mathrm{dm}^{3}$, and the types of waters were referred to as "mine water I, II, III", respectively. The particles sizes below $0.5 \mathrm{~mm}$ were floated in saline water which content of $\mathrm{NaCl}$ was $117 \mathrm{mg} / \mathrm{dm}^{3}$. The products of flotation (concentrates and tailings) were analysed for the ash content in accordance with PN-ISO 1171: 2002.

All flotation experiments were conducted with the procedure described below. The specified coal samples were weighed, then process water with specific salt content was added, and the coal was soaked for 10 minutes. Then, a hexanol frother was added. Hexanol is an aliphatic alcohol and in the flotation process it performs the collecting and frothing properties sine it adsorbs on both solid-liquid and liquid-gas phases. It results in increasing hydrophobicity of coal and reducing the speed at which air bubbles flow to the surface. In addition, it allows to form a durable flotation froth, ensuring stabilization of air bubbles and preventing their coalescence.

At specific pulp density, the dose of flotation reagent was specified as $0.08 \mathrm{~g}$ of hexanol per $1 \mathrm{dm}^{3}$ of solution, which corresponds to consumption of $1142 \mathrm{~g}$ per $1 \mathrm{Mg}$ of dry feed. To ensure reagent adsorption, the flotation machine was started and the solution was mixed for 3 minutes without air [6]. The air was added to the flotation tank after the set time and the fraction flotation was performed. The concentrates were collected at time: $15,15,30,30,30$, every $60 \mathrm{~s}$. The total flotation time was 6 min. The concentrates and tailings were dried at temperature of $60^{\circ} \mathrm{C}$. The obtained results allowed calculating the basic indices to estimate the flotation process effectiveness, namely concentrate $\left(\gamma_{\mathrm{k}}\right)$ and tailings $\left(\gamma_{\mathrm{o}}\right)$ yields, ash content in individual upgrading products $(\beta$-concentrate, $v-$ tailings, $\alpha-$ feed), and the recovery of combustibles and volatile matter in the concentrate $\left(\varepsilon_{\mathrm{k}}\right)$ as well as in the tailing $\left(\varepsilon_{\mathrm{o}}\right)$. 


\section{Results and discussion}

\subsection{Presentation of results}

Tables 1 and 2 include the calculation results limited to yield $(\gamma)$, recovery of combustible and volatile in concentrates $\left(\varepsilon_{\mathrm{k}}\right)$. The flotation kinetics curves were plotted in order to evaluate the upgrading progress in time. Figures 1 and 2 present the empirical recovery vs time relationships, respectively for particle size classes $0.315-0.2$ and $0.1-0.063 \mathrm{~mm}$. The upgrading curves for individual particle size classes were plotted to illustrate the flotation effectiveness (Figures 3 and 4).

Table 1. Selected coal flotation parameters.

Particie size 0,315-0,2 mm

\begin{tabular}{|c|c|c|c|c|c|c|c|c|c|c|c|c|}
\hline \multirow{2}{*}{$\begin{array}{l}\text { time } \\
t[\mathrm{~s}]\end{array}$} & \multicolumn{3}{|c|}{ distilled water } & \multicolumn{3}{|c|}{ Mine water I } & \multicolumn{3}{|c|}{ Mine water II } & \multicolumn{3}{|c|}{ Mine water III } \\
\hline & $\begin{array}{l}\Sigma \gamma \downarrow \\
{[\%]}\end{array}$ & $\beta[\%]$ & $\varepsilon[\%]$ & $\begin{array}{c}\Sigma \gamma \\
\downarrow[\%]\end{array}$ & $\beta[\%]$ & $\varepsilon[\%]$ & $\begin{array}{l}\Sigma \gamma \downarrow \\
{[\%]}\end{array}$ & $\beta[\%]$ & $\varepsilon[\%]$ & $\begin{array}{l}\Sigma y \downarrow \\
{[\%]}\end{array}$ & $\beta[\%]$ & $\varepsilon[\%]$ \\
\hline 0 & 0.00 & 0.00 & 0.00 & 0.00 & 0.00 & 0.00 & 0.00 & 0.00 & 0.00 & 0.00 & 0.00 & 0.00 \\
\hline 15 & 4.90 & 5.13 & 4.80 & 9.54 & 18.67 & 9.73 & 7.48 & 25.37 & 7.57 & 9.77 & 33.36 & 8.34 \\
\hline 30 & 8.07 & 5.07 & 7.92 & 11.48 & 17.99 & 11.80 & 10.72 & 26.86 & 10.63 & 14.54 & 29.12 & 13.19 \\
\hline 60 & 12.39 & 4.86 & 12.18 & 15.63 & 14.49 & 16.75 & 14.71 & 24.91 & 14.98 & 18.80 & 26.25 & 17.74 \\
\hline 90 & 17.00 & 4.60 & 16.76 & 18.12 & 14.36 & 19.44 & 17.96 & 25.13 & 18.23 & 22.56 & 23.74 & 22.01 \\
\hline 120 & 19.88 & 4.67 & 19.59 & 20.89 & 13.94 & 22.52 & 21.20 & 25.61 & 21.38 & 25.31 & 22.50 & 25.10 \\
\hline 180 & 26.51 & 4.27 & 26.23 & 26.14 & 13.52 & 28.33 & 26.68 & 25.82 & 26.84 & 31.08 & 20.85 & 31.47 \\
\hline 240 & 32.28 & 4.19 & 31.96 & 30.29 & 13.15 & 32.97 & 31.42 & 24.44 & 32.19 & 35.34 & 21.08 & 35.69 \\
\hline 300 & 38.62 & 3.96 & 38.33 & 34.72 & 12.70 & 37.98 & 35.16 & 23.44 & 36.51 & 39.10 & 19.91 & 40.07 \\
\hline 360 & 44.09 & 4.02 & 43.74 & 40.25 & 12.10 & 44.33 & 38.65 & 22.44 & 40.65 & 43.61 & 18.82 & 45.30 \\
\hline tailing & 100.00 & 3.24 & 100.00 & 100.00 & 20.20 & 100.00 & 100.00 & 26.26 & 100.00 & 100.00 & 21.85 & 100.00 \\
\hline
\end{tabular}

Table 2. Selected coal flotation parameters.

Particie size ( 0,1 -0,063) mm

\begin{tabular}{|c|c|c|c|c|c|c|c|c|c|c|c|c|}
\hline time & \multicolumn{3}{|c|}{ distilled water } & \multicolumn{3}{|c|}{ Mine water I } & \multicolumn{3}{|c|}{ Mine water II } & \multicolumn{3}{|c|}{ Mine water III } \\
\hline$t[\mathbf{s}]$ & $\begin{array}{l}\Sigma \gamma \downarrow \\
{[\%]}\end{array}$ & $\beta[\%]$ & $\varepsilon[\%]$ & $\Sigma \gamma \downarrow[\%]$ & $\beta[\%]$ & $\varepsilon[\%]$ & $\begin{array}{l}\Sigma y \downarrow \\
{[\%]}\end{array}$ & $\beta[\%]$ & $\varepsilon[\%]$ & $\begin{array}{l}\Sigma y \downarrow \\
{[\%]}\end{array}$ & $\beta[\%]$ & $\varepsilon[\%]$ \\
\hline 0 & 0.00 & 0.00 & 0.00 & 0.00 & 0.00 & 0.00 & 0.00 & 0.00 & 0.00 & 0.00 & 0.00 & 0.00 \\
\hline 15 & 42.47 & 15.57 & 49.77 & 39.19 & 17.18 & 46.97 & 35.42 & 16.20 & 45.38 & 34.13 & 15.17 & 44.42 \\
\hline 30 & 57.05 & 15.19 & 67.16 & 50.90 & 17.39 & 60.84 & 48.19 & 17.15 & 61.04 & 45.44 & 15.41 & 58.97 \\
\hline 60 & 67.19 & 15.66 & 78.66 & 59.98 & 18.06 & 71.12 & 57.83 & 18.05 & 72.45 & 55.95 & 18.43 & 70.02 \\
\hline 90 & 71.32 & 16.13 & 83.02 & 63.32 & 18.45 & 74.73 & 61.93 & 18.70 & 76.97 & 63.89 & 21.10 & 77.34 \\
\hline 120 & 75.75 & 16.82 & 87.46 & 66.19 & 19.33 & 77.27 & 65.30 & 19.52 & 80.34 & 66.47 & 22.09 & 79.45 \\
\hline 180 & 78.61 & 17.29 & 90.24 & 68.94 & 19.96 & 79.85 & 71.08 & 21.19 & 85.64 & 74.80 & 26.11 & 84.80 \\
\hline 240 & 79.87 & 17.53 & 91.43 & 70.85 & 20.59 & 81.41 & 74.94 & 22.34 & 88.97 & 77.58 & 26.91 & 87.00 \\
\hline 300 & 80.03 & 17.56 & 91.58 & 72.16 & 21.18 & 82.31 & 76.87 & 22.71 & 90.83 & 79.76 & 27.52 & 88.70 \\
\hline 360 & 81.30 & 17.93 & 92.62 & 73.00 & 21.42 & 83.00 & 78.80 & 23.19 & 92.53 & 81.15 & 27.96 & 89.70 \\
\hline tailing & 100.00 & 27.95 & 100.00 & 100.00 & 30.90 & 100.00 & 100.00 & 34.59 & 100.00 & 100.00 & 34.82 & 100.00 \\
\hline
\end{tabular}




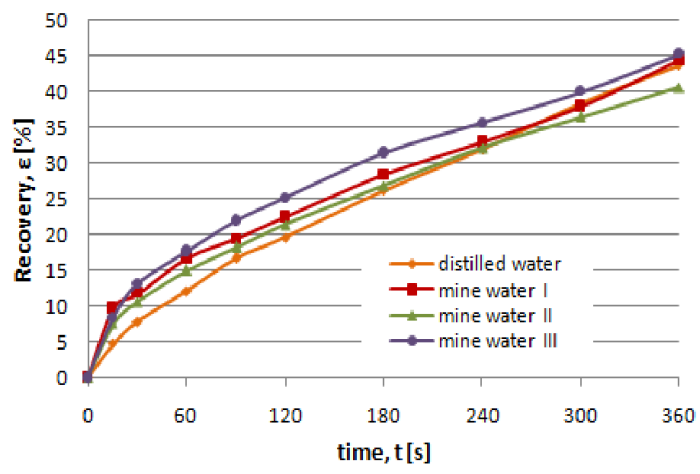

Figure 1. Flotation kinetics curves in waters with different salinity for particle size $0.315-0.2 \mathrm{~mm}$.

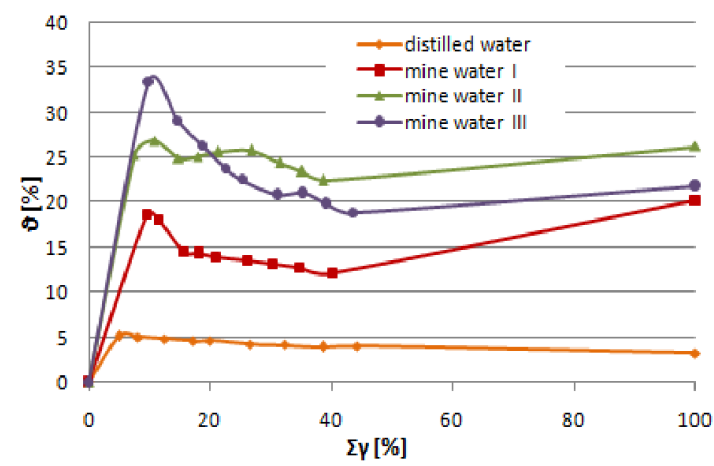

Figure 3. Changes of ash content in concentrate from, yield particle size $0.315-0.2 \mathrm{~mm}$.

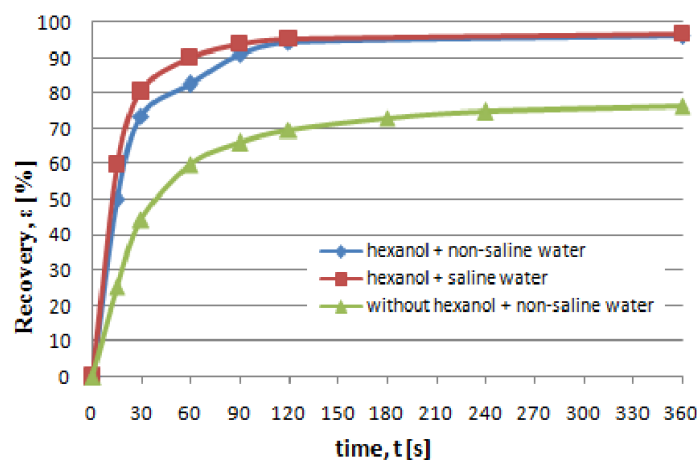

Figure 5. Recovery vs. time.

The graphical interpretation of flotation results for the below $0.5 \mathrm{~mm}$ grain size is performed by plotting the flotation kinetics curves (Fig. 5) and the curves indicating the changes of ash content in concentrates (Fig. 6) and in tailings (Fig. 7).

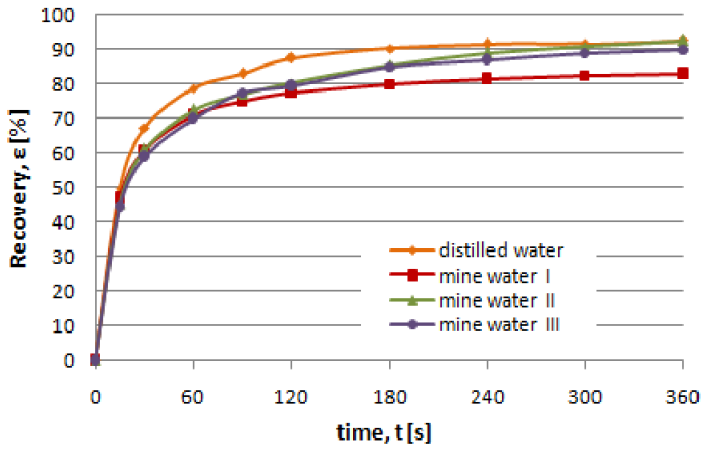

Figure 2. Flotation kinetics curves in waters with different salinity for particle size $0.1-0.063 \mathrm{~mm}$.

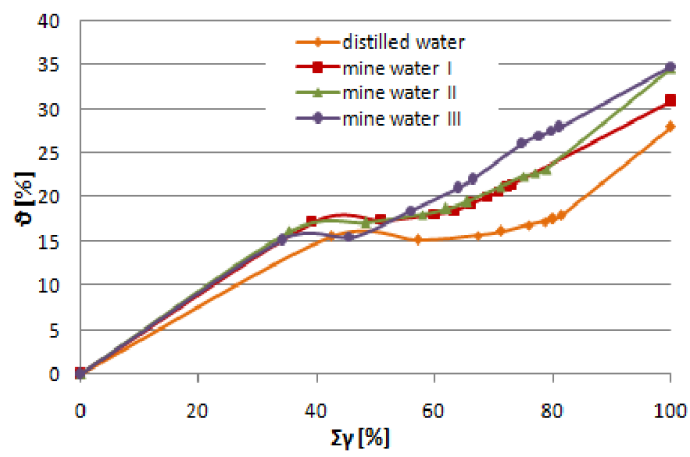

Figure 4. Changes of ash content in concentrate from yield, particle size 0.1-0.063 mm.

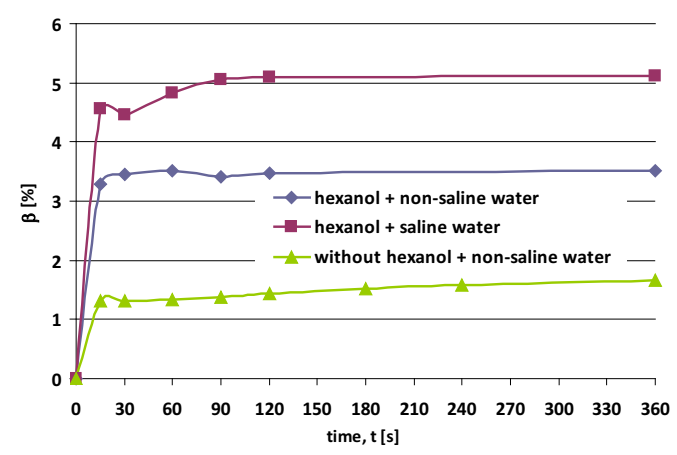

Figure 6. Changes of ash content in concentrate from time. 


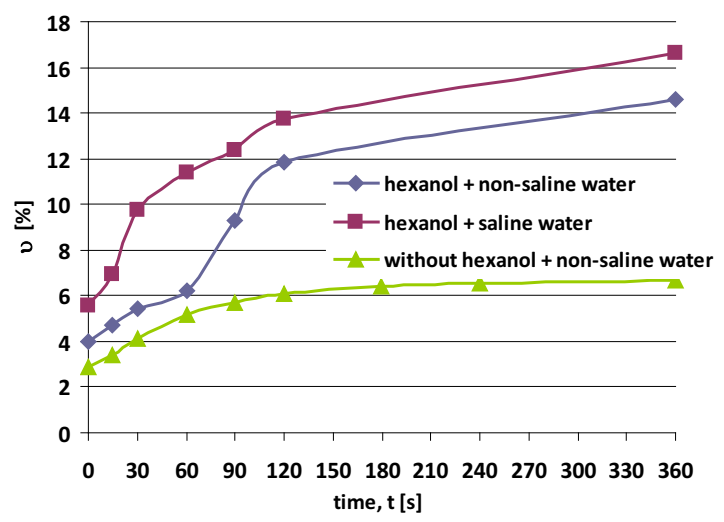

Figure 7. Changes of ash content in tailing with time.

\subsection{Discussion}

The analysis of results presented in Table 1 and shape of curves in Fig. 1 indicate a constant rising trend for recovery values with time. The shape of kinetics curves suggests that the upgrading process could continue because the balance recovery value (a plateau on the plot) was not reached. The indicated shape of kinetics curves should be expected in the case of highly effective flotation [7]. The course of flotation process is similar for each type of water used as shown by the shape of curves, and the highest intensity is observed in the first 3 minutes of flotation. The review of conditions improving the upgrading effectiveness indicates that in the most saline water (mine water III) flotation reaches the best values of yield, recovery and concentrate parameters. This proves that the presence of $\mathrm{NaCl}$ enhances the flotation process.

It is also evident that the concentrate yield in the first 15 seconds of the process is the lowest in thecase of distilled water $(4.90 \%)$, whereas for the mine water I it reaches $9.54 \%$, mine water II $7.48 \%$, and mine water III $-9.77 \%$. The yield increases and also recovery increases by $1 / 3$ or even $1 / 2$ in comparison with flotation in distilled water. It clearly indicates a positive impact of the presents of salt on the properties of solids subjected to flotation and also an improvement of gaseous phase properties under such conditions $[7,8]$.

Since inder industrial conditions narrow particle size classes are not upgraded, the successive analyses include the results of flotation tests conducted for particle below $0.5 \mathrm{~mm}$. The analysis of results shown in Fig. 6 indicates that the greatest process speed for two flotation process with hexanol was obtained in the first 2 minutes, and the process without hexanol stabilized in the $3^{\text {rd }}$ minute when $69.73 \%$ concentrate yield was achieved in flotation without reagent. Almost identical result, with difference of about $1 \%$ was detected in flotation in saline and non-saline water with addition of hexanol. It should be noted that both process with hexanol were similar, and the kinetics curves were practically identical. It would suggest similar properties of phases remaining in contact during flotation [6]. Only from 15 to 105 seconds of the process, the obtained recovery results differed by about $10 \%$ in each successive concentrate collection. An initial recovery increase was observed for floatation in saline water, but it was later reduced. It is shown in the plot as another convergence of curves in the same point (about 95\% concentrate recovery). The final result was the $96 \%$ recovery for flotation with hexanol in both the $\mathrm{NaCl}$ saline water and non-saline water. The recovery of flotation without reagent was however significantly lower and reached $76.29 \%$. It is evident that hexanol increased the coal flotation effectiveness and at the same time it can be stated that water salinity facilitates the uplift of coal grains. The ash content in the concentrate in the non-saline water without hexanol varies in the $1.31-1.66 \%$ range, in the $3.28-3.51 \%$ range in non-saline water with hexanol, and in the $4.56-5.12 \%$ range in saline water with hexanol. 
In order to evaluate the flotability of the tested material, the experimented results were presented in the form of Henry curves (Fig. 9). The ideal upgrading is denoted when on the Henry curve is the relationship between the yield and the content of useful ingredient is a horizontal line [10]. If the relationship is like this, then we obtain only concentrate containing pure useful components. When the upgrading line is vertical and the content of useful components remains constant and equal to $\alpha$, then there is no upgrading. The analysis of curves presented in Fig. 8 and 9 indicates that the hexanol in this case gives high concentrate yields in both saline and non-saline water. The concentrate yield reached $95 \%$ in pure water flotation, and $90 \%$ in the process in the saline water. Such yield values substantiate the statement that the coal floated with addition of hexanol becomes easily upgradeable.

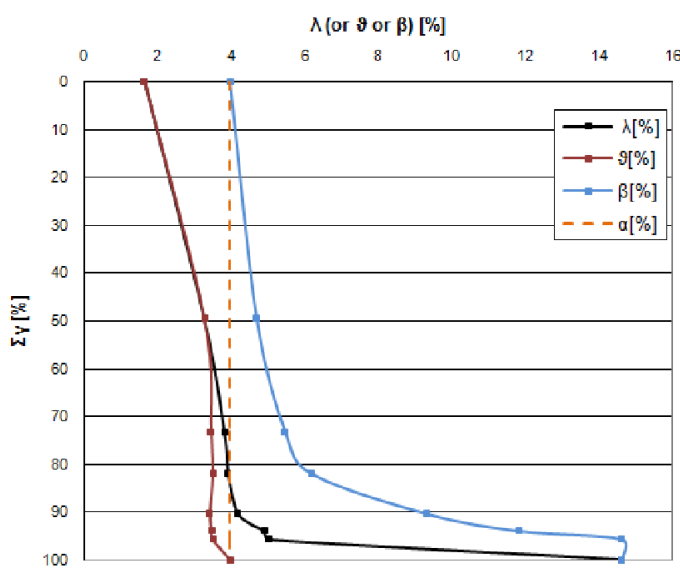

Figure 8. Washability curves in non-saline water.

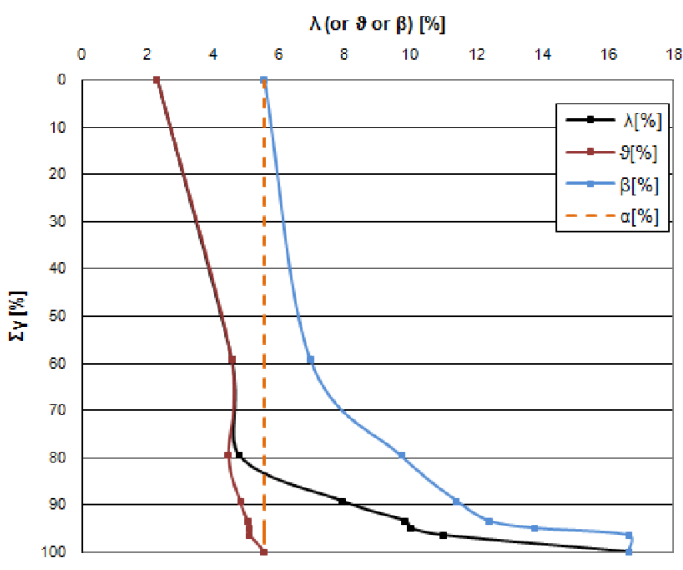

Figure 9. Washability curves in saline water.

\subsection{Summary}

In the light of literature on the flotation upgrading in the presence of inorganic substances, mainly salt, it seems appropriate to distinguish two terms: salt flotation conducted under conditions of increased surface tension in relation to water, and flotation conducted in the presence of salt in the pulp where the surface tension is reduced. The process conducted in the presence of salt is accompanied by increased froth stability, which rises as the concentration of frothing agent increases.

The froth stability is also very closely related to the bubble size, namely: the smaller the bubbles, the more stable the froth. This conclusion should be verified for each frothing agent used. The froth stability in coal flotation and the increased content of $\mathrm{NaCl}$ in process water improves the flotation effectiveness, with maximum recovery equal to $90 \%$. In addition, it should be noted that high flotation effectiveness depends on the feed particle size. Although narrow particle size classes are not upgraded in industrial conditions, a verification of process effectiveness for small particle size ranges allows to forecast their behaviour in real conditions. Hence, on the basis of the presented results one should expect the process effectiveness of $40-90 \%$ recovery when upgrading the $0.315-.0063 \mathrm{~mm}$ grain size class in saline water. Thus, coal flotation in saline waters brings benefits: (i) conscious control of upgrading to increase its effectiveness, and (ii) reduced consumption of reagents which means lower costs. And most importantly, it allows the mines to recirculate the process water. The results of laboratory experiments prove that using an aliphatic alcohol, in both non-saline and saline waters, gives the concentrate yield in excess of $90 \%$. This type of reagent improves the effectiveness of coal slurry flotation, and water salinity facilitates floating the coal particles to the froth product. In addition, hexanol adsorbing on the surface of bubbles creates durable flotation aggregates, thus increasing the probability of floating the coal particles. The application of this flotation reagent gives high ash content in tailings, in excess of $16 \%$ in saline water, with the ash content in concentrates not 
exceeding $6 \%$. The purpose of coal upgrading is to maximize the content of combustible and volatile matter and to minimize the ash content in concentrates. Such results are guaranteed by the use of hexanol which has an apolar carbon chain. In addition, it should be noted that:

(i) the process effectiveness is much better in the case of upgrading materials with a wider particle size range than for narrow ranges floated separately. It gives much higher process effectiveness and good quality upgrading products. Wider particle size class and lower content of solids in the flotation pulp allow to reduce the reagent consumption, giving the better upgrading results;

(ii) the excessive salt content in process water causes a transfer of a significant amount of salt to the products of upgrading, thus increasing its yields and simultaneously reducing the technological parameters;

(iii) use of saline mine waters reduces the costs of technological processes by limiting the demand for fresh water and improves the environmental protection by reducing the amount of mine wastewater discharged to rivers.

\section{Acknowledgement}

This work was done as a part of the University of Science and Technology Research Program No.11.11.100.276 and was used in the MSc dissertations.

\section{References}

1. R.H.Yoon, Min. Cong. J., 68, 76 (1982)

2. R.H. Yoon, J.B.Sabey, Interfacial Phenomena in Coal Technology, G.D. Botsaris, G.D., Glazman, Y.M. (Eds.) (New York, 87 1989).

3. J.S.Laskowski, Coal Flotation and Fine Coal Utilisation (Elsevier, Amsterdam, 2001) R.H. Yoon, J.B.Sabey, Interfacial Phenomena in Coal Technology, G.D. Botsaris, G.D., Glazman, Y.M. (Eds.) (New York, 87 1989).

4. O. Ozdemir, E. Taran, M.A. Hampton, S.I. Karakashev, A.V. Nguyen, Surface Process., 92, 177 (2009)

5. A. Mlynarczykowska, A. Grabiec, A. Krawczykowska, D. Saramak, Mineral Processing Conference 2013 (Zakopane, 2013)

6. M. Brozek, A. Mlynarczykowska, Kinetics of flotation,( Press. IGSMiE PAN, 2009)A

7. A. Rodrigo, T, Graua, J.S. Laskowski, K. Heiskanen, Int. J. Miner. Process., 76, 225 (2005)

8. M. Brozek, A. Mlynarczykowska, Physicoch. Prob. Miner. Process., 44, 23 (2012)

9. J. Drzymala, , Mineral Processing, (Oficyna Wydawnicza Politechniki Wrocławskiej, 2001)

10. L. Wang, X. Qu, Miner. Eng., 26, 50 (2012) 